\title{
Dielectric properties of colon polyps, cancer, and normal mucosa: Ex vivo measurements from 0.5 to $20 \mathrm{GHz}$
}

\author{
Marta Guardiola ${ }^{a)}$ \\ BCN-MedTech, Department of Information and Communication Technologies, Universitat Pompeu Fabra, Barcelona 08018, Spain
}

Santiago Buitrago

CommSensLab, Department of Signal Theory and Communications, Universitat Politècnica de Catalunya, Barcelona 08034, Spain

Glòria Fernández-Esparrach

Endoscopy Unit, Institut de Malalties Digestives i Metabòliques, IDIBAPS, CIBERehd, Hospital Clínic, Universitat de Barcelona, Barcelona 08036, Spain

Joan M. O'Callaghan and Jordi Romeu

CommSensLab, Department of Signal Theory and Communications, Universitat Politècnica de Catalunya, Barcelona 08034, Spain

Miriam Cuatrecasas

Pathology Department, CDB, Hospital Clínic, IDIBAPS, Universitat de Barcelona Banc de Tumors Biobanc Clinic-IDIBAPS,

Barcelona 08036, Spain

Henry Córdova

Endoscopy Unit, Institut de Malalties Digestives i Metabòliques, IDIBAPS, CIBERehd, Hospital Clínic, Universitat de Barcelona, Barcelona 08036, Spain

Miguel Ángel González Ballester

BCN-MedTech, Department of Information and Communication Technologies, Universitat Pompeu Fabra, Barcelona 08018, Spain ICREA, Barcelona 08010, Spain

\section{Oscar Camara}

BCN-MedTech, Department of Information and Communication Technologies, Universitat Pompeu Fabra, Barcelona 08018, Spain

(Received 11 October 2017; revised 9 May 2018; accepted for publication 18 May 2018; published 15 June 2018)

Purpose: Colorectal cancer is highly preventable by detecting and removing polyps, which are the precursors. Currently, the most accurate test is colonoscopy, but still misses $22 \%$ of polyps due to visualization limitations. In this paper, we preliminary assess the potential of microwave imaging and dielectric properties (e.g., complex permittivity) as a complementary method for detecting polyps and cancer tissue in the colon. The dielectric properties of biological tissues have been used in a wide variety of applications, including safety assessment of wireless technologies and design of medical diagnostic or therapeutic techniques (microwave imaging, hyperthermia, and ablation). The main purpose of this work is to measure the complex permittivity of different types of colon polyps, cancer, and normal mucosa in ex vivo human samples to study if the dielectric properties are appropriate for classification purposes.

Methods: The complex permittivity of freshly excised healthy colon tissue, cancer, and histological samples of different types of polyps from 23 patients was characterized using an open-ended coaxial probe between 0.5 and $20 \mathrm{GHz}$. The obtained measurements were classified into five tissue groups before applying a data reduction step with a frequency dispersive single-pole Debye model. The classification was finally compared with pathological analysis of tissue samples, which is the gold standard.

Results: The complex permittivity progressively increases as the tissue degenerates from normal to cancer. When comparing to the gold-standard histological tissue analysis, the sensitivity and specificity of the proposed method is the following: $100 \%$ and $95 \%$ for cancer diagnosis; $91 \%$ and $62 \%$ for adenomas with high-grade dysplasia; $100 \%$ and $61 \%$ for adenomas with low-grade dysplasia; and $100 \%$ and $74 \%$ for hyperplastic polyps, respectively. In addition, complex permittivity measurements were independent of the lesion shape and size, which is also an interesting property comparing to current colonoscopy techniques.

Conclusions: The contrast in complex permittivities between normal and abnormal colon tissues presented here for the first time demonstrate the potential of these measurements for tissue classification. It also opens the door to the development of a microwave endoscopic device to complement the outcomes of colonoscopy with functional tissue information. (C) 2018 American Association of Physicists in Medicine [https://doi.org/10.1002/mp.13016] 
Key words: colorectal cancer, Debye model, dielectric characterization, ex vivo tissues, microwave imaging

\section{INTRODUCTION}

Colorectal cancer (CRC) is a serious and increasing health problem in countries with a Westernized lifestyle where, in average, affects 1 in 20 men and 1 in 30 women over $50 \mathrm{yr}$ old. ${ }^{1}$ Worldwide CRC is the third most common cancer and the fourth most deadly one just behind lung, liver, and stomach cancer. ${ }^{2}$ In average, the 5 -yr survival rate is $65 \%$, however, it is highly dependent on the stage of diagnosis. Fortunately, CRC is a disease that can be cured in more than $90 \%$ of patients if detected at an early stage. ${ }^{3}$ The development of CRC is characterized by an ordered series of events that are referred to as adenoma-carcinoma sequence: healthy mucosa develops dysplasia and forms small polyps, called adenomas, which may grow and eventually become adenocarcinomas ${ }^{4}$ unless resected. ${ }^{5}$ Several systematic reviews of the literature have shown that CRC screening is cost-effective and prospective studies with a large number of cases demonstrate a reduction in CRC risk during follow-up patients undergoing colonoscopy screening, ${ }^{6}$ which is the standard in clinical routine. Besides colonoscopy, there are other methods for CRC screening such as stool-based tests, double-contrast barium edema, and CT colonography. ${ }^{7}$ If there is suspicion of polyps in these tests, a colonoscopy will be needed. ${ }^{7}$

To date, colonoscopy is the most effective diagnostic and therapeutic technique for the prevention of CRC, since it allows the identification of polyps with a relatively good accuracy. It is also the only method able to remove tissue samples (e.g., polyps) in the entire colon for subsequent histological analysis. Several prospective studies demonstrate that colonoscopy with polypectomy (removal of polyps) reduces the incidence of CRC by $40-90 \%{ }^{8,9}$ Nevertheless, colonoscopy is far from being perfect: $22 \%$ of polyps are not detected ${ }^{10}$ and the risk of cancer after a negative colonoscopy is still $7.9 \% .^{11}$ The main cause of this lack of efficacy is the visualization limitation ${ }^{12}$ of the optical camera placed at the tip of the endoscope. Studies indicate that $13.4 \%$ of the colon surface area might not be visualized during a standard colonoscopy ${ }^{13}$ due to reduced field of vision $\left(<180^{\circ}\right)$, inhomogeneous illumination, colon angulations and folds, and poor cleaning. Another limitation of colonoscopy is the subjectivity in the assessment of several characteristics of the lesions such as size, position, and tissue classification, which highly depends on the experience of the endoscopist. The final diagnosis is subsequently based on the results of the histological analysis of the resected tissues, which is considered the gold standard. As a security measure, current clinical guidelines ${ }^{3}$ therefore recommend removing all lesions found in a colonoscopy study, including small lesions $(<5 \mathrm{~mm})$ that are often benign polyps. A pathologist then analyses all lesions and emits a histology report. This clinical workflow is labor- and time-intensive, making the processing of these patient data inefficient and significantly increasing the burden for the healthcare system.

In recent years, several devices and technologies have been developed to improve the detection rate of polyps such as high-definition endoscopes, endoscopes with multiple lenses (retrovision capability), and mucosal flattening accessories. ${ }^{14}$ Advances in computer vision may also help in the detection of morphological changes in colon images, such as shape, color, or texture, ${ }^{15}$ which may indicate the presence of an abnormality. The current trend in colonoscopy is to provide additional information during the exploration (often called in situ diagnosis) to help doctors in their decision-making. For example, magnification ${ }^{14}$ of the image may help to detect morphological changes; electronic chromoendoscopy narrow band imaging may also enhance the visualization of the vascularization abnormalities related to cancer. ${ }^{16}$ However, all these techniques exploit the same contrast mechanism based on the optical image, thus being restricted to information visualized with the camera at the tip of the endoscope. To overcome this limitation a different contrast mechanism is needed. Some methods such as near infrared Raman ${ }^{17}$ or terahertz spectroscopy ${ }^{18}$ have also been proposed for cancer diagnosis purposes. However, there is not a technique yet that can distinguish between benign and malignant lesions during the study.

Microwave imaging has the potential to complement conventional colonoscopy to improve both polyp detection rate and in situ tissue classification. Microwaves can generate images without restriction of the field of view $\left(360^{\circ}\right)$ and offer a fair trade-off between resolution and light opaque tissue penetration, ${ }^{19}$ therefore, potentially reducing visualization problems of conventional colonoscopy. In addition, microwaves can provide a quantitative differentiation of normal and abnormal tissues based in their dielectric properties. ${ }^{20}$ Finally, the technology is safe (low power and nonionizing radiation) and portable. For these reasons microwave imaging is a promising method for population screening, diagnosis and monitoring of cancer patients.

Microwave imaging tries to evaluate objects hidden or embedded in a structure (or medium) using electromagnetic (EM) waves in the regime of the microwaves. In the field of medicine, microwaves have been established as a good solution for treatment of cancer (hyperthermia and ablation) ${ }^{21}$ and for monitoring of vital signs. ${ }^{22}$ Furthermore, there is ongoing research studying microwave imaging for imagebased diagnosis in an extensive number of applications: acute ischemia or cerebral hemorrhage, ${ }^{23}$ pulmonary edema, urinary incontinence, osteoporosis, ${ }^{24}$ and breast cancer detection $^{25,26}$; some of these studies are already in clinical phases. ${ }^{27}$ As far as we know, microwave imaging has never been proposed before for endoscopic applications. 
To assess the feasibility of microwave imaging for CRC diagnosis it is crucial to have good dielectric contrast between healthy colon, different types of polyps, and cancer tissues over the frequency band of interest. It has previously been demonstrated that the complex permittivity of some cancer and healthy tissues change noticeably. ${ }^{20}$ However, to the best of our knowledge, there is no available data for normal and abnormal colon tissues in humans over $900 \mathrm{MHz}$. Done-Sik et al. ${ }^{28,29}$ measured the complex permittivity of colon cancer and healthy colon of nude mice over $0.2-5 \mathrm{GHz}$ band, while Joines et al. ${ }^{30}$ reported the complex permittivity of healthy and malignant colon human samples between 50 and $900 \mathrm{MHz}$ band. Gabriel et al. extended the healthy colon measurements to the $0.5-20 \mathrm{GHz}$ band using ovine models. ${ }^{31-33}$

In this paper, we present data from dielectric properties measured on ex vivo human samples of different types of colon polyps and healthy colon tissues, which was not available in the literature. Measurements on dielectric properties were acquired using the open-ended coaxial probe method across the frequency range 0.5 to $20 \mathrm{GHz}$. With these data we formed five groups of tissues based on the similarities in their dielectric properties. Subsequently, we reduced the data using a Debye model. The remainder of this paper is organized as follows. Section 2 describes the methodology for obtaining tissue samples, conducting dielectric spectroscopy and data analysis. Section 3 presents the results obtained from the human tissue study. Section 4 discusses the applicability of dielectric property data to colonoscopy tests. Finally, Section 5 summarizes the main conclusions of this study.

\section{MATERIALS AND METHODS}

This work was performed at the Endoscopy Unit of Hospital Clínic of Barcelona (HCB), after being approved by the Ethical Committee of Clinical Investigation at HCB. All patients signed an informed consent permitting colorectal examinations and use of their data for research purposes. A total of 23 patients undergoing screening colonoscopies, polypectomies, and surgical colectomies were enrolled in the study. Between one and seven specimens per patient were collected, leading to a total of 59 freshly excised samples of different histological types of polyps, healthy colon, and CRC tissue (Table I), thus providing a complete overview of the colon pathology. The measurement protocol was nondestructive for subsequent pathological analysis of the same sample and was designed to reduce the manipulation of the sample and the time between excision and measurement.

In the remaining of this section, we first present colon cancer disease and the existing tissue classification methods used in daily clinical routine. We then describe the protocol followed to measure the complex permittivity of freshly excised colon samples using a precision open-ended coaxial probe. A statistical analysis was carried out to assess the quality of the obtained measurements and the corresponding tissue classification based on dielectric data. We finally explain the fitting of the measured data to a single-pole Debye model.
TABLE I. Morphology, size, and number of polyps by malignant potential (LGD, HGD, are low- and high-grade dysplasia, respectively) and total.

\begin{tabular}{lcccr}
\hline \hline Morphology & Size $(\mathrm{mm})$ & LGD & HGD & N \\
\hline Tubular adenoma & $6-25$ & 16 & 6 & 22 \\
Villous adenoma & 7 & 0 & 1 & 1 \\
Tubulovillous adenoma & $25-60$ & 2 & 0 & 2 \\
Hyperplastic & $3-12$ & - & - & 8 \\
Sessile serrated & 18 & - & - & 5 \\
\hline \hline
\end{tabular}

\section{A. Classification of samples}

Colon polyps are slow-growing overgrowths of the colonic mucosa originating from superficial glandular epithelial cell lining in the colon and rectum, and generally protruding into the lumen. When a polyp degenerates into cancer it may infiltrate into the wall (invasive). Colon polyps can be classified according to their histological and shape characteristics, as following. Histology defines tissue and cell anatomy based on its appearance under the microscope and sets the grade of dysplasia (i.e., abnormality of development or cell anomaly of growth and differentiation). Depending on the grade of dysplasia, polyps can be diagnosed as either neoplastic or non-neoplastic, if they have lost its normal differentiation or not, respectively. Hyperplastic polyps are the most common type of non-neoplastic or benign proliferations of cells. Neoplastic lesions or adenomas can be categorized according to their histological appearance into: tubular, villous, or tubulovillous, ${ }^{34}$ and according to their grade of dysplasia. Adenomas with low-grade dysplasia (LGD) are more similar to healthy mucosa, while malignant adenomas can present high-grade dysplasia (HGD) or have already degenerated to adenocarcinoma. ${ }^{35}$ Recently, serrated variants of polyps have been considered as a new category of neoplastic polyps because of their malignant potential. ${ }^{36,37}$ Figures 1 and 2 present pictures of five different colon tissue samples and their corresponding histology images, respectively. Histology images show the tissue composition and shape characteristics of the cells under study. Normal mucosa [see Fig. 2(a)] is the innermost layer of colon wall, which is composed by epithelial cells that form glandular crypts, and connective tissue (called lamina propria) filling the empty spaces. Polyps are also composed by epithelial cells but with certain modifications: for example, hyperplastic polyps [Fig. 2(b)] present a serrated and dilated contour of the glandular crypts; tubular adenomas [Fig. 2(c)] show elongated glandular crypts and cellular proliferation. Figure 2(d) shows the adenoma-carcinoma sequence in the same sample. An infiltrating adenocarcinoma is originated on a tubular adenoma that presents an in situ adenocarcinoma in the middle. Finally, Fig. 2(e) shows an invasive adenocarcinoma infiltrating the muscular layer.

The shape of polyps is usually categorized during a colonoscopy exploration using the standard Paris classification, ${ }^{38}$ as can be seen in Fig. 3. This classification method divides the polyps into protruded (e.g., pedunculated, Ip; sessile, Is), 

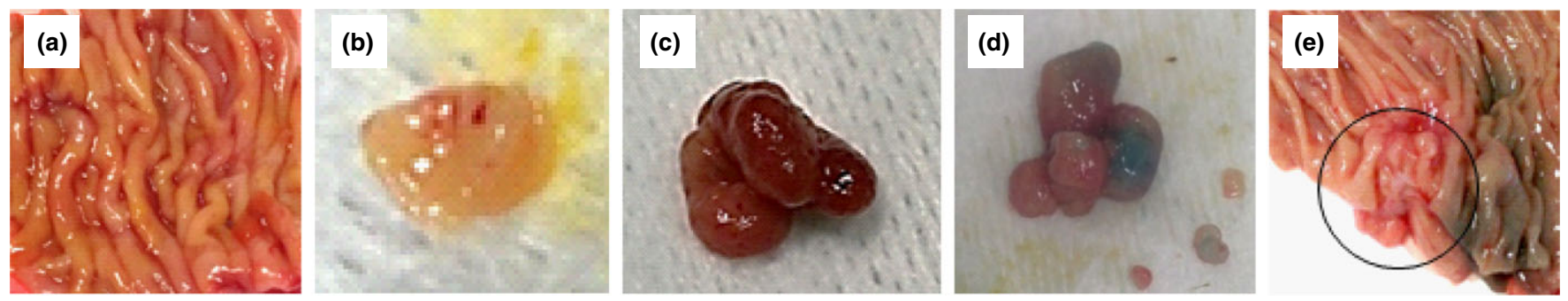

FIG. 1. Pictures of five tissue specimens including: (a) healthy mucosa; (b) hyperplastic polyp of $8 \mathrm{~mm}$; (c) tubular adenoma with low-grade dysplasia of $12 \mathrm{~mm}$; (d) tubular adenoma with high-grade dysplasia of $25 \mathrm{~mm}$; and (e) colon mucosa with an adenocarcinoma of $15 \mathrm{~mm}$ marked with a circle. [Color figure can be viewed at wileyonlinelibrary.com]
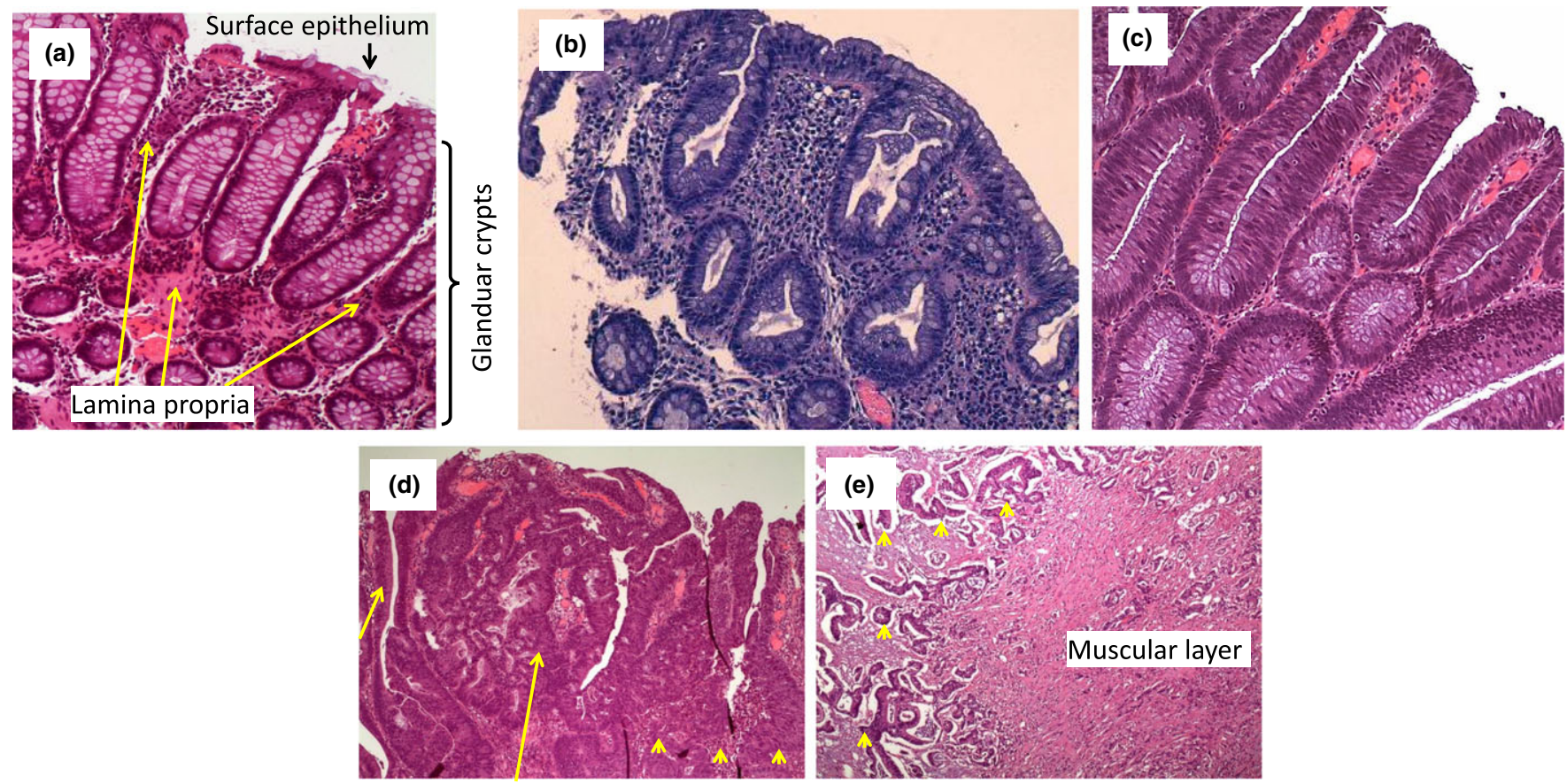

FIG. 2. (a) Normal mucosa composed of surface epithelium, glandular crypts, and lamina propria. (b) Hyperplastic polyp showing a serrated and dilated contour of the glandular crypts. (c) Tubular adenoma with low-grade dysplasia is similar to normal mucosa but presents increased proliferation of nuclei in the elongated crypts. (d) Adenoma-carcinoma sequence shown in the same lesion: residual tubular adenoma (left-short arrow) evolving into an in situ adenocarcinoma in the middle (center-long arrow), and to an infiltrating adenocarcinoma (right arrowheads). (e) Infiltrating adenocarcinoma with epithelial glands (arrowheads) invading the muscular layer of the colon wall. [Color figure can be viewed at wileyonlinelibrary.com]

Protruded

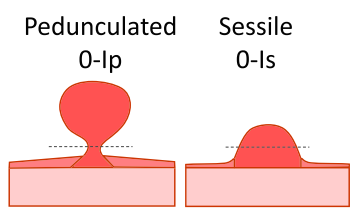

\section{Non-protruded}

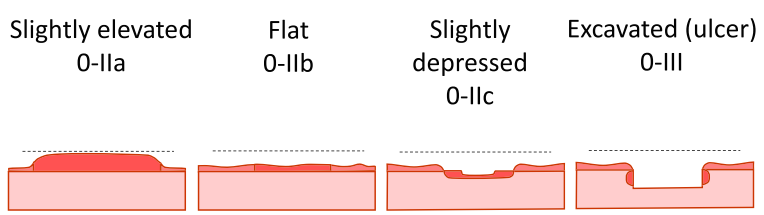

FIG. 3. Morphological categories of polyps based on the standard Paris classification. The dotted line represents twice the thickness of the mucosa layer. ${ }^{38}$ [Color figure can be viewed at wileyonlinelibrary.com]

and nonprotruded (e.g., slightly elevated, IIa; flat, IIb; slightly depressed, IIc; excavated, III). We measured 9 pedunculated, 11 sessile, and 18 slightly elevated polyps. In general, small size and nonprotruded polyps are the ones that report higher polyp miss rates. ${ }^{39}$
For this study, we used freshly excised colon samples from 23 patients, from which we analyzed 38 polyps with different characteristics, as summarized in Table I; 11 samples of cancer and 10 samples of normal colon mucosa. 


\section{B. Measurement setup and calibration experiments}

The complex permittivity of polyps and cancer was measured using a Keysight 85070 E dielectric probe kit with an open-ended high temperature coaxial probe ${ }^{40}$ connected to an Agilent E8362B vector network analyzer (VNA), as can be seen in Fig. 4. The complex permittivity measurements of the samples were recorded at room temperature using a linear frequency range between 0.5 and $20 \mathrm{GHz}$. The number of measured frequency points was 101. The samples were placed on a stand with a scissor lift controlled manually using a leadscrew. The probe was fixed on the axis of a manual positioner and connected to the VNA with a cable. The cable was attached to the same axis with a zip tie to reduce sagging and remained untouched during the entire measurement. The stand was manually lifted until the entire probe aperture made firm contact with the sample. In this way, the coaxial probe was perfectly firm, being easier to avoid air gaps and control the pressure. The VNA was calibrated before each measurement using the common three standard loads (open, short, and load). Deionized water was used as the load in the calibration process. The dimensions of the probe head imposed size and homogeneity restrictions to the sample. As a reference, Table II shows the probe range of usability provided by the manufacturer.

Before starting measurements on human samples, the appropriateness of the coaxial probe method for colon tissue measurement was verified. We performed several experiments with different materials with known complex permittivity to determine the accuracy, minimum size of the sample (lateral dimensions and thickness), and repeatability.

\section{B.1. Accuracy}

We measured the complex permittivity of several materials such as $0.051 \mathrm{M}$ aqueous $\mathrm{NaCl}$ solution $^{41}$ and methanol ${ }^{42}$ (liquids with high and low relative permittivity and conductivity values, respectively). Subsequently, the measured complex permittivity was compared with the theoretical values.

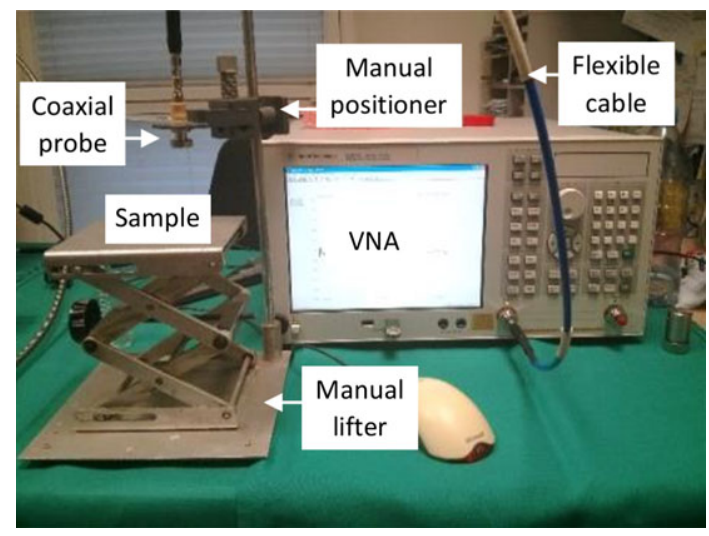

FIG. 4. Setup for dielectric spectroscopy. The open-ended coaxial probe was fixed on a manual positioner and connected to a vector network analyzer (VNA). [Color figure can be viewed at wileyonlinelibrary.com]
TABLE II. Main features and requirements of the Keysight 85070E high-temperature probe.

Frequency range

$200 \mathrm{MHz}-20 \mathrm{GHz}$

Maximum relative permittivity of the sample

$\epsilon^{\prime}<1000$

Minimum loss tangent of the sample

$\tan \delta>0.05$

Minimum sample diameter

$20 \mathrm{~mm}$

Minimum sample thickness

$\sqrt{\epsilon^{\prime}} \mathrm{mm}$

Maximum granule size

$0.3 \mathrm{~mm}$

\section{B.2. Minimum sample size}

The minimum thickness of the sample required to obtain reliable measurements was evaluated using a mixture of deionized water and methanol in a certain proportion to obtain a similar complex permittivity as the average of colon tissues. We poured the mixture in a beaker placed on a metal surface and we measured the complex permittivity for different levels of mixture to assess the minimum thickness of the sample. The minimum lateral size of the sample was assessed directly with the measured complex permittivity data of the excised tissues (between 5 and $30 \mathrm{~mm}$ in diameter). The minimum lateral size is largely dictated by the probe's geometry. Since the interrogating field is mostly confined between the outer and the inner conductor of the probe, the sample must cover completely this area to provide accurate measurements. The effects of different sample sizes at $10 \mathrm{GHz}$ were statistically analyzed using the tissues classified as adenoma with LGD because it was the most populated group.

\section{B.3. Repeatability}

We took three measurements of each sample in different spots to track the effects, if any, of the sample orientation, homogeneity, and exerted pressure.

\section{C. Clinical protocol and human sample experiments}

In human sample experiments, we registered details on sample preparation, measurement times, and the temperature, to analyze if they had any impact on the measured complex permittivity. The clinical protocol, shown in Fig. 5, was defined in close collaboration with the medical staff to optimize the integration of the electromagnetic measurements into the daily clinical practice and reduce the time between excision and measurement. The time between excision and measurement was between $2 \mathrm{~min}$ and $2 \mathrm{~h}$ depending on the type of tissue and how the samples were obtained, as follows.

- Polyps: Nearly all polyps and superficial cancers could be resected endoscopically by routine polypectomy methods, including cautery or cold snare removal. ${ }^{43}$ Cold snare technique involves capturing the lesion with a snare wire and then closing it to cut the lesion. Cautery can also be used to destroy residual polyp tissue. In these procedures, a liquid solution 


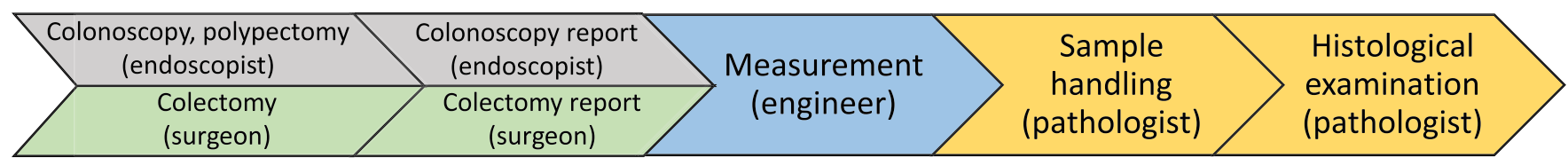

FIG. 5. Clinical protocol for the acquisition of electromagnetic measurements within daily medical routine. [Color figure can be viewed at wileyonlinelibrary.c om]

was commonly injected under the polyp to vertically separate the lesion from the deeper colon layers and reduce the chances of perforation. Endoscopists traditionally use saline, often with a blue dye, succinylated gelatin, or adrenaline. In our measurements, the distribution of different solutions in the studied samples was: adrenaline in $32 \%$ of samples, succinylated gelatin in $34 \%$ of samples, indigo carmine dye in $44 \%$ of samples (some samples contained more than one additive). Measurements took place at the Endoscopy Unit of $\mathrm{HCB}$, in an adjoining room to the colonoscopy boxes where the screening colonoscopies and polypectomies were performed. Immediately after the excision from the patient, the sample was transported to the room where we measured the complex permittivity. Just after the measurement, the samples were introduced in a formalin solution and were sent to the Pathology Department for histological analysis. Characteristics of the polyps (size, Paris classification, location into the colon, resection technique, and additives injected) were described in a written report. Measurements were quickly obtained for these samples after the excision, around 2 min later. For this reason, we did not take any action to prevent tissue dehydration.

- Nonpolyp tissues: in clinical routine, when it is not possible to fully remove a polyp, the patient is referred to colectomy (surgical procedure to remove all or parts of the colon). For our study, healthy mucosa and invasive cancer tissues were obtained from colectomies performed in an operation room. Cancer tissue properties were measured from the available large pieces (e.g., entire colon of $1.5 \mathrm{~m}$ long, $10 \mathrm{~cm}$ width, and $1 \mathrm{~cm}$ of thickness; or half a colon). Healthy mucosa properties were also measured from the same colon pieces. The samples were not available till the end of the operation, with a delay between $30 \mathrm{~min}$ and $2 \mathrm{~h}$. The specimen was kept as long as possible inside the patient to prevent tissue dehydration. Just after the surgery, the resected colon was transported to the Pathology Department where we measured the complex permittivity of tissues before being handled by the pathologist. We relied on the pathologist indications to select the most meaningful area for our measurements. For all the examined tissues we received a histology report of the lesion that was added to the colonoscopy report.

Another important issue was the sample homogeneity. Since the measured complex permittivity represents the average value of all tissues present in the sensing area, ${ }^{44}$ an homogeneous sample is desirable. Usually, when surgery is conducted to remove abnormal tissues, a margin of safety (e.g., healthy tissue) is also excised in order to ensure all abnormal tissue is fully removed. Polyps with high-grade dysplasia were removed with a safety margin of $1 \mathrm{~mm}$. Since dysplastic lesions are generally large, it was easy to select a measurement area avoiding the margins of healthy tissue with the help of the clinicians. Contrarily, non-neoplastic polyps were removed without leaving a margin of healthy tissue, as recommended in colonoscopy guidelines.

\section{D. Complex permittivity data fitting}

The complex permittivity, $\epsilon^{*}(\omega)=\epsilon^{\prime}(\omega)-j \epsilon^{\prime \prime}(\omega)$, is composed by a real part, $\epsilon^{\prime}$, referred to as relative permittivity, and an imaginary part, $\epsilon^{\prime \prime}$, often expressed in terms of conductivity, $\sigma=\epsilon_{0} \epsilon^{\prime \prime} \omega(\mathrm{S} / \mathrm{m})$, or loss tangent, $\tan \delta=\epsilon^{\prime \prime} / \epsilon^{\prime}$, which accounts for the losses $\left(\epsilon_{0}=8.85 \cdot 10^{-12} \mathrm{~F} / \mathrm{m}\right.$ is the free space permittivity and $\omega=2 \pi f$, where $f$ is the frequency in $\mathrm{Hz}$ ).

Single and multipole Debye $\mathrm{e}^{45,46}$ and Cole-Cole models $^{20,31,32,47,48}$ have been proposed in the literature to parameterize the measured values of the complex wideband permittivity of biological tissues at microwave frequencies. Debye models have recently gained interest because they require less computational overhead ${ }^{46}$ than Cole-Cole methods in the largely utilized Finite-Difference Time-Domain (FDTD) numerical methods. ${ }^{48}$ For an optimal representation of the data, the number of poles in both models should correspond to the number of dielectric relaxations of the tissue over the investigated frequency range. ${ }^{31,32}$ Within our frequency range (from 0.5 to $20 \mathrm{GHz}$ ), two relaxations can be identified as follows: gamma dispersion occurs with a center frequency near $25 \mathrm{GHz}$ at body temperature due to the dipolar relaxation of water; and a small dispersion, often called delta or UHF (Ultra High Frequency) dispersion, occurs between 0.1 and $3 \mathrm{GHz}^{26,49,50}$ in biological tissues. In some studies, ${ }^{32}$ a higher order model is used because it provides more flexibility to achieve a better fit to the data. However, if the number of poles is too large, model parameters may be fitted to noise variations and not to the data itself. Another aspect that influences the parametric model fitting quality is the frequency spacing of the measured data. Accordingly, having a sufficient number of data points around the relaxation contributes to a more accurate modeling of the measured data, especially at low frequencies. ${ }^{51}$ Our measurements content fewer samples in the lower frequency range than in the higher range. Therefore, we decided to accommodate our data above $2.5 \mathrm{GHz}$ to a single-pole Debye 
model. For sake of comparison, we also calculated the parametric values for a two-pole Debye model and a single-pole Cole-Cole model, ${ }^{32}$ using the following equations:

$$
\begin{aligned}
& \epsilon^{*}(\omega)=\epsilon_{\infty}+\sum_{i=1}^{N} \frac{\Delta \epsilon_{i}}{1+j \omega \tau_{i}}+\frac{\sigma_{s}}{j \omega \epsilon_{0}} \\
& \epsilon^{*}(\omega)=\epsilon_{\infty}+\frac{\Delta \epsilon}{1+(j \omega \tau)^{1-\alpha}}+\frac{\sigma_{s}}{j \omega \epsilon_{0}}
\end{aligned}
$$

Equation (1) is the multipole Debye model, where $N$ is the number of poles. The magnitude of the dispersion, $\Delta \epsilon=\epsilon_{s}-\epsilon_{\infty}$, is given by the difference between the static permittivity $\left(\epsilon_{s}\right.$, limiting value of the relative permittivity obtained at alternating field frequencies where $\omega \tau \ll 1$ ) and the permittivity at infinite frequency $\left(\epsilon_{\infty}, \omega \tau \ll 1\right)$; $\tau$ is the time constant; and $\sigma_{s}$ the static ionic conductivity. The ColeCole model [Eq. (2)] incorporates a distribution parameter $(\alpha)$ to model the broadening of the dispersion. ${ }^{32}$

We used the genetic algorithm (GA) available in the Matlab optimization toolbox (MATLAB and Optimization Toolbox Release 2012b, The MathWorks, Inc., Natick, MA, USA) to fit the measured relative permittivity and conductivity values over the band of interest to the three parametric models. ${ }^{46}$ The genetic algorithm minimizes an objective/error function, which in our case it computes the differences between the experimental data and the Debye model, ${ }^{46}$ giving an estimate of the quality of the fitting:

$$
\text { Error }=\frac{\sum_{k=1}^{N}\left[\frac{\epsilon^{\prime}\left(\omega_{k}\right)-\epsilon_{d}^{\prime}\left(\omega_{k}\right)}{\operatorname{median}\left[\epsilon_{d}^{\prime}(\underline{\omega})\right]}\right]^{2}+\sum_{k=1}^{N}\left[\frac{\sigma\left(\omega_{k}\right)-\sigma_{d}\left(\omega_{k}\right)}{\operatorname{median}\left[\sigma_{d}(\underline{\omega})\right]}\right]^{2}}{N}
$$

where $\epsilon_{d}$ and $\sigma_{d}$ are the relative permittivity and conductivity calculated with the Debye formulation [Eq. (1)] at each individual measured angular frequency $\omega_{k} . \epsilon^{\prime}, \sigma$ are the measured relative permittivity and conductivity, respectively, and $\underline{\omega}$ the vector of all frequencies of interest used in the fitting procedure. The genetic algorithm repeatedly modifies a population of individual solutions. An individual $x$ is a set of four Debye parameters $\left[\epsilon_{\infty}, \Delta \epsilon, \tau, \sigma_{s}\right]$ to which the objective function is applied. The optimization starts from a population of 50 randomly generated individuals represented by a $50 \times 4$ matrix. At each step, the genetic algorithm randomly selects individuals from the current population and uses them as parents to produce the children for the next generation (crossover fraction of 0.8 , Gaussian mutation with zero mean and standard deviation equal to 1). Over successive generations (set to a maximum of 1000), the population evolves toward an optimal solution minimizing the objective function. In our case, the optimization is subjected to a linear constraint of the form $A x \leq b$ to ensure that $\omega \tau \ll 1$, as suggested by Mustafa et al. ${ }^{46}$ Bound value ranges were applied to each of the parameters in order to ensure that the GA did not converge toward local optima. ${ }^{51}$ Commonly the algorithm terminates when either a maximum number of generations is produced or when the average relative change in the best fitness function value over generations is less than or equal to the tolerance $\left(1 \cdot 10^{-10}\right)$.

The chi-square goodness of fit test is used to decide whether there is any difference between the fitted data and the expected (measured) data. The test is defined $a^{51}$ :

$$
\chi^{2}=\sum_{i=1}^{n} \frac{\left(O_{i}-E_{i}\right)^{2}}{E_{i}}
$$

where $O_{i}$ is the fitted data, $E_{i}$ is the expected or measured data, and $n$ is the total number of frequency data points. Small values of the chi-square goodness of fit test indicate that there are not significant differences between the fitted and expected value.

\section{E. Statistical analysis}

We performed a statistical analysis to study the effects of sample size, injected solutions, resection method, and polyp shape on the measured complex permittivity. For the analysis, we used the relative permittivity and conductivity values at $5 \mathrm{GHz}$ of all the samples categorized as adenoma with LGD because it was the largest group. We also analyzed if the complex permittivity data could be used to univocally classify the different colon tissues. To this aim, a one-way nested analysis of variance (ANOVA) $F$-test was performed using the SPSS statistics software (IBM Corp. Released 2010. Version 19.0. Armonk, NY, USA).

The performance of a decision criterion is often characterized in terms of sensitivity and specificity. The sensitivity, also called the true positive (TP) rate, measures the percentage of cases having a polyp or cancer that are correctly diagnosed as having the lesion. A false negative (FN) occurs when a negative result is reported to a patient that does have a lesion. The specificity, also called the true negative (TN) rate, measures the percentage of healthy cases that are correctly identified as not having any polyp or cancer. A false positive (FP) is reported when the test wrongly indicates that a lesion is present. The values of sensitivity and specificity are related to TP, FP, TN, and FN values through the following formulas ${ }^{52}$ :

$$
\text { Sensitivity }=\frac{T P}{T P+F N} \quad \text { Specificity }=\frac{T N}{T N+F P}
$$

\section{RESULTS}

\section{A. Calibration experiments}

\section{A.1. Accuracy}

Results obtained from the calibration experiments showed that the open-ended coaxial method was adequate for our measurements. Figure 6 represents the accuracy of the acquired measurements for $0.051 \mathrm{M}$ aqueous $\mathrm{NaCl}$ solution at $25^{\circ} \mathrm{C}$, pure methanol, and a mixture having similar complex permittivity to colon tissues ( $\epsilon^{\prime}$ around 45 at $5 \mathrm{GHz}$ ). 

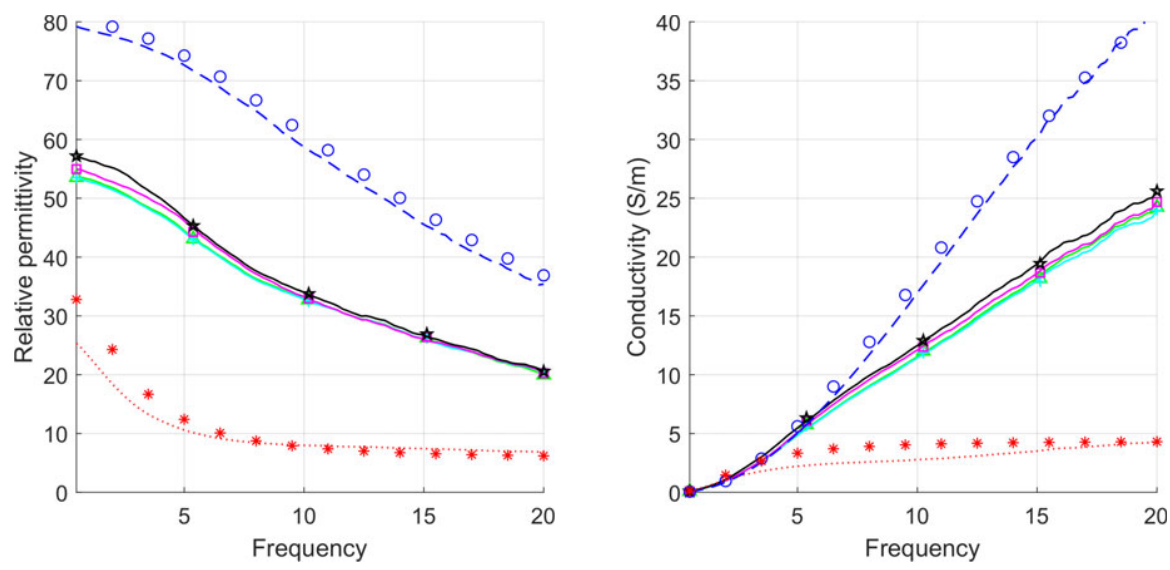

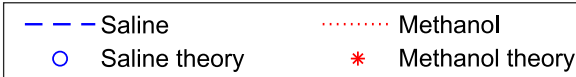

$\begin{gathered}\triangle \operatorname{Mix} 20 \mathrm{~mm} \\ -\operatorname{Mix} 15 \mathrm{~mm}\end{gathered}-\operatorname{Mix} 6 \mathrm{~mm}$

FIG. 6. Accuracy of dielectric property measurements (relative permittivity, left; conductivity, right) for $10 \mathrm{~mm}$ of saline solution at $25^{\circ} \mathrm{C}$ poured in a beaker, $10 \mathrm{~mm}$ of methanol and a mixture poured in different levels $(4,6,15$, and $20 \mathrm{~mm})$. A comparison between measured and theoretical values for the saline solution and methanol is provided. [Color figure can be viewed at wileyonlinelibrary.com]

Both relative permittivity and conductivity values of these liquids show an excellent agreement with the corresponding theoretical values ${ }^{41,42}$ with an error lower than $5 \%$ in all cases.

\section{A.2. Minimum sample size}

We used a mixture of liquids ( $\epsilon^{\prime}=45$ at $5 \mathrm{GHz}$ ) to experimentally confirm the minimum measurable thickness of the sample. According to the manufacturer, the thickness of the sample must be greater than $\sqrt{45}=6.7 \mathrm{~mm}$. Figure 6 shows the complex permittivity measured for different amounts of mixture in a beaker $(4,6,15$, and $20 \mathrm{~mm})$. The complex permittivity does not vary for quantities of mixture greater than $15 \mathrm{~mm}$. Below this level, the complex permittivity begins to change slightly. An error lower than a $5 \%$ for both the relative permittivity and the conductivity is obtained for mixture levels greater than $6 \mathrm{~mm}$. The effect of the sample size was analyzed with an ANOVA $F$-test. We compared the $P$-values of adenomas with LGD of all sizes with adenomas with LGD larger than $10 \mathrm{~mm}$. We found statistical differences $(P \leq 0.05)$ due to the size when we considered all the samples $(P=0.003$ for the relative permittivity and $P=0.0001$ for the conductivity), while there were no significant differences when considering samples larger or equal to $10 \mathrm{~mm}$ ( $P=0.425$ for the relative permittivity and $P=0.441$ for the conductivity). Therefore, only samples larger than $10 \mathrm{~mm}$ in diameter accomplished the size requirements. After this selection we obtained 51 valid measurements (32 polyps, 10 adenocarcinomas, 9 normal colon mucosa).

\section{A.3. Repeatability}

The orientation of the sample did not produce differences in the measured complex permittivity within the same tissue. Pressure over the tissue during the experiments was controlled to avoid any effect on the obtained measurements.

\section{B. Human sample experiments}

We also analyzed the effect of the resection technique and the injected solutions within the adenoma with LDG group. None of the solutions produced a statistically significant effect neither in the relative permittivity nor the conductivity, with $P$-values above the significance threshold (the following couple of values per solution correspond to the relative permittivity and the conductivity): indigo carmine dye, $P=0.596$ and 0.407 ; succinylated gelatin, $P=0.802$ and $P=0.133$; adrenaline, $P=0.650$ and $P=0.336$. We did not expect any influence of the chosen solution on the measurements since they were injected underneath the lesion before its removal; for this reason, traces of substances should not remain inside the sample. The resection method (cautery and cold snare) did not cause significant changes in complex permittivity either $(P=0.8267$ and $P=0.2587$ for the relative permittivity and conductivity, respectively). It was expected that the resection method would not influence the measurements since the snare is closed at the base of the polyp, while the polyp itself remains intact. Cautery is only applied to remove large polyps and mainly affects the surrounding colon mucosa. To ensure that cautery is not influencing our measure, we selected the measurement area as far as possible from the cauterization zone.

Using the data provided in the histology report, we identified and labeled the valid measured samples. We obtained 9 samples of healthy colon mucosa, 32 samples of different histological types of polyps, and 10 adenocarcinomas. The majority of measured samples (52\%) were adenomas since their size was adequate for the probe. Contrarily, hyperplastic polyps were scarce $(12 \%)$ because they were generally small (less than $5 \mathrm{~mm}$ ) and had to be excluded due to the probe size requirements. Healthy mucosa $(17 \%)$ and large adenocarcinomas $(19 \%)$ were only obtained from surgical colectomies. Moreover, we could only measure healthy mucosa from left colon colectomies as the right colon wall was too thin (only 
$2 \mathrm{~mm}$ thick) to fulfill coaxial probe thickness requirements, especially at lower frequencies.

\section{C. Complex permittivity of polyps, healthy mucosa, and adenocarcinomas}

Using the data provided in the pathology report, we identified and labeled the valid measurements as: adenocarcinomas, healthy mucosa, hyperplastic polyps, tubular adenomas with LGD, tubular adenoma with HGD, villous adenomas with HGD, and tubulovillous adenomas with HGD. Figure 7 shows the plots of resulting relative permittivity and conductivity. Visual observation of this figure allows identifying three groups of curves that present differentiated dielectric properties: benign tissues (healthy mucosa and hyperplastic polyps) and polyps with LGD occupy the lowest cold-colored part; adenomas with HGD, the intermediate magenta part; and adenocarcinomas, the upper red part. This suggests a relationship between the complex permittivity of the samples and the grade of dysplasia, for example, higher values of both the relative permittivity and conductivity for adenocarcinomas and polyps with HGD. Based on these observations, we finally classified the samples into five groups: adenocarcinomas, adenomas with HGD, adenomas with LGD, hyperplastic polyps, and healthy colon mucosa, respectively. Statistical analysis confirmed that this classification produces significant differences between groups $(P=0.017$ for the relative permittivity and $P=0.050$ for the conductivity) and nonsignificant differences within groups. Polyp shape or texture did not produce changes on relative permittivity values $(P=0.582)$. Figure 8 shows the median values (solid lines) and the standard deviation (shaded bands, 95\% confidence interval) of the relative permittivity and conductivity of the five tissue groups. It can be observed that the relative permittivity and conductivity of adenocarcinomas and benign tissues are clearly differentiated between 3 and $10 \mathrm{GHz}$. Likewise, adenocarcinomas and adenomas with HGD can be clearly distinguished, while adenomas with LGD overlap with benign tissues. Therefore, the complex permittivity represents clearly the dysplasia grade of the adenomas: the higher the complex permittivity the higher the probability of malignancy.

To examine the detection capacity of adenocarcinomas, the percentage differences between the relative permittivity and conductivity of adenocarcinomas and the rest of the analyzed tissues (adenoma with HGD, adenoma with LGD, hyperplastic polyps, and healthy mucosa) were calculated at multiple frequencies, as shown in Figs. 9(a) and 9(b). There was an average difference of $20-30 \%$ in relative permittivity between cancer and benign tissues for frequencies between 2 and $8 \mathrm{GHz}$, which tended to decrease as the frequency increased. The larger differences in conductivity values were found between 5 and $8 \mathrm{GHz}$ (around 30-60\%). Between cancer and adenomas with HGD, the differences were more stable over the frequency range (15\% and $25 \%$ for the relative permittivity and conductivity, respectively, at $7 \mathrm{GHz}$ ). Figures 9(c) and 9(d) show the percentage differences between the relative permittivity and conductivity of healthy mucosa vs all lesions for the sake of comparison with conventional colonoscopy. The contrast between healthy mucosa and cancer is $30-100 \%$, and between healthy mucosa and adenoma with HGD is $10-57 \%$, which confirms the capacity of the complex permittivity, especially the conductivity, to identify dysplastic tissues.

A complementary analysis of the accuracy of dielectric properties for tissue classification can be done from sensitivity and specificity values. Table III shows the sensitivity and specificity to detect the different histological types of polyps and adenocarcinomas from the rest of the colon tissues. The sensitivity and specificity were calculated for both the relative permittivity and the conductivity at different frequencies. Note that the performance depends on the frequency as anticipated in Fig. 9. It can be observed that the sensitivity to detect adenocarcinomas is $100 \%$ for all the frequencies
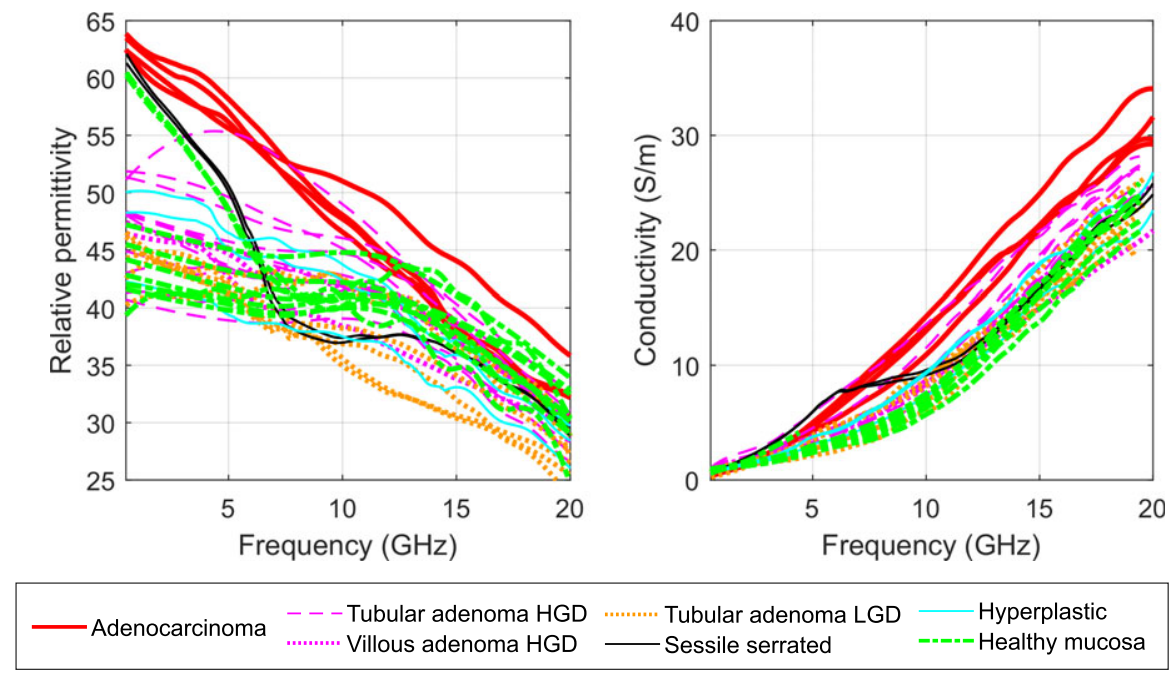

FIG. 7. Measured relative permittivity and conductivity of the different types of colon tissues. HGD and LGD stand for high-grade dysplasia and low-grade dysplasia, respectively. [Color figure can be viewed at wileyonlinelibrary.com] 

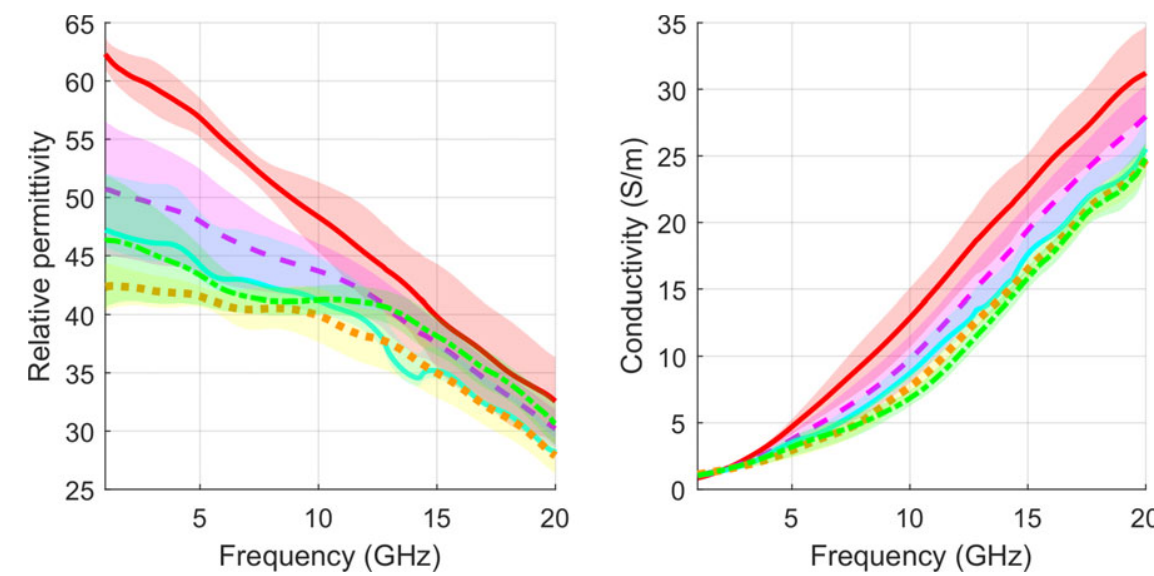

\begin{tabular}{ll}
\hline Adenocarcinoma & $-\cdots "-$ Adenoma LGD \\
--- Adenoma HGD & Hyperplastic
\end{tabular}

FIG. 8. Measured relative permittivity and conductivity of colon tissues. The lines represent the median values and the colored areas around the median show the 95\% confidence interval. HGD and LGD stand for high-grade dysplasia and low-grade dysplasia, respectively. [Color figure can be viewed at wileyonlinelibra ry.com]

(a) 70

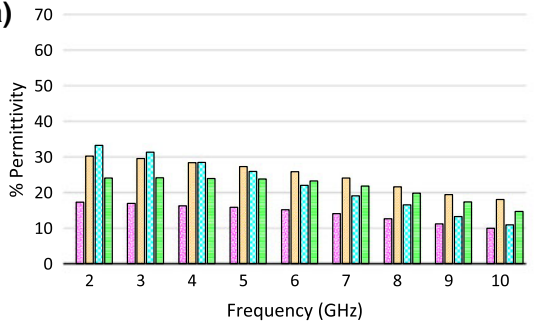

(c)

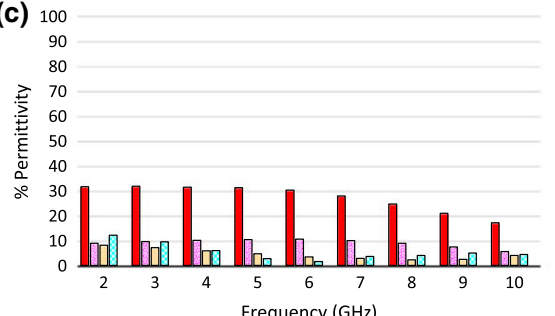

(b) 70

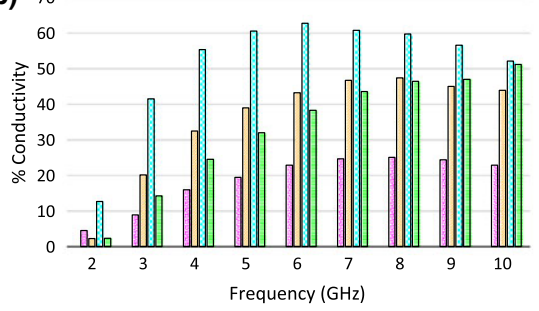

(d) 100

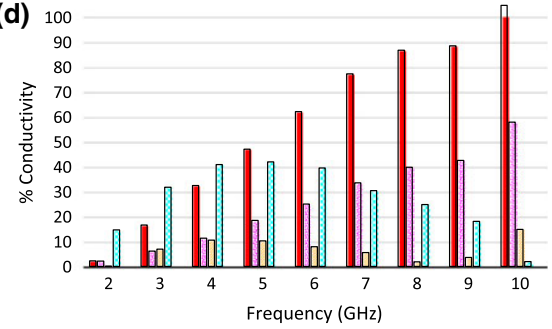

Adenocarcinoma

图 Adenoma HGD

$\square$ Adenoma LGD

Hyperplastic

目 Healthy mucosa

Fig. 9. Percentage differences on complex permittivity of adenocarcinoma (top) and healthy mucosa (bottom) vs the remaining types of tissues for different frequencies. HGD and LGD stand for high-grade dysplasia and low-grade dysplasia, respectively. [Color figure can be viewed at wileyonlinelibrary.com]

analyzed, while the specificity is maximum $(95.12 \%)$ between 5 and $8 \mathrm{GHz}$ using the relative permittivity data. For the adenomas with HGD, the maximum sensitivity $(90.91 \%)$ is reached using the conductivity data at 7 and $8 \mathrm{GHz}$ and the specificity using both the relative permittivity and conductivity data is $61.76 \%$ at $9 \mathrm{GHz}$. For the adenomas with LGD a perfect sensitivity is obtained at $5 \mathrm{GHz}$ using the relative permittivity data and the specificity using the relative permittivity data is $60.61 \%$ at 5 and $7 \mathrm{GHz}$. Finally, for the hyperplastic polyps a perfect sensitivity is obtained at all the frequencies analyzed and the maximum specificity using the relative permittivity data is $73.68 \%$ at $9 \mathrm{GHz}$. For all the lesions, the highest values of sensitivity and specificity using the conductivity are achieved using higher frequencies
(9 GHz), while the highest performance using the relative permittivity is obtained at $5 \mathrm{GHz}$. In general, the frequency that offers a better trade-off between sensitivity and specificity is $5 \mathrm{GHz}$.

\section{D. Data fitting and reduction}

We calculated the parameters of Cole-Cole, single-, and two-pole Debye models of the five colon tissue groups from the median measured complex permittivity over $2.5-20 \mathrm{GHz}$. To quantify the quality of the parametric models, the fitting error [Eq. (3)] and the chi-squared values ${ }^{51}$ were estimated, as presented in Table IV. It can be observed from the results that the single-pole Debye model provides the smallest fitting 
TABLE III. Sensitivity (Sn) and specificity (Sp) for the relative permittivity $(\epsilon)$ and the conductivity $(\sigma)$ in the diagnosis of each lesion: adenocarcinoma (ACA), adenoma with high-grade dysplasia (A-HGD), adenoma with low-grade dysplasia (A-LGD), and hyperplastic polyps (HP) vs the rest of tissues for different frequencies. The largest values among all frequencies are marked in bold.

\begin{tabular}{|c|c|c|c|c|c|c|c|c|c|c|c|c|}
\hline Tissue & $\epsilon^{\prime} \mathrm{Sn}$ & $\epsilon^{\prime} \mathrm{Sp}$ & $\sigma \mathrm{Sn}$ & $\sigma \mathrm{Sp}$ & $\epsilon^{\prime} \mathrm{Sn}$ & $\epsilon^{\prime} \mathrm{Sp}$ & $\sigma \mathrm{Sn}$ & $\sigma \mathrm{Sp}$ & $\epsilon^{\prime} \mathrm{Sn}$ & $\epsilon^{\prime} \mathrm{Sp}$ & $\sigma \mathrm{Sn}$ & $\sigma \mathrm{Sp}$ \\
\hline A-HGD & 81.28 & 61.76 & 72.73 & 58.82 & 90.91 & 55.88 & 72.73 & 58.82 & 81.82 & 55.88 & 90.91 & 58.82 \\
\hline A-LGD & 90.76 & 60.61 & 75.00 & 39.39 & 100 & 60.61 & 83.33 & 42.42 & 91.67 & 60.61 & 75.00 & 39.39 \\
\hline HP & 71.43 & 52.63 & 100 & 10.53 & 100 & 13.16 & 100 & 10.53 & 100 & 7.89 & 100 & 10.53 \\
\hline & \multicolumn{4}{|c|}{$7 \mathrm{GHz}$} & \multicolumn{4}{|c|}{$8 \mathrm{GHz}$} & \multicolumn{4}{|c|}{$9 \mathrm{GHz}$} \\
\hline Tissue & $\epsilon^{\prime} \mathrm{Sn}$ & $\epsilon^{\prime} \mathrm{Sp}$ & $\sigma \mathrm{Sn}$ & $\sigma \mathrm{Sp}$ & $\epsilon^{\prime} \mathrm{Sn}$ & $\epsilon^{\prime} \mathrm{Sp}$ & $\sigma \mathrm{Sn}$ & $\sigma \mathrm{Sp}$ & $\epsilon^{\prime} \mathrm{Sn}$ & $\epsilon^{\prime} \mathrm{Sp}$ & $\sigma \mathrm{Sn}$ & $\sigma \mathrm{Sp}$ \\
\hline ACA & 100 & 95.12 & 100 & 85.37 & 100 & 95.12 & 100 & 85.37 & 100 & 92.68 & 100 & 90.24 \\
\hline A-HGD & 72.73 & 61.76 & 90.91 & 58.82 & 81.82 & 58.82 & 90.91 & 58.82 & 81.81 & 53.87 & 90.91 & 61.76 \\
\hline
\end{tabular}

TABLE IV. Comparison of the fitting error and chi-squared values $\left(\chi^{2}\right)$ for the relative permittivity $\left(\epsilon^{\prime}\right)$ and conductivity $(\sigma)$ of the single-pole Debye model, single-pole Cole-Cole model, and two-pole Debye model, respectively.

\begin{tabular}{|c|c|c|c|c|c|c|c|c|c|}
\hline Tissue & \multicolumn{3}{|c|}{ Single-pole Debye } & \multicolumn{3}{|c|}{ Two-pole Debye } & \multicolumn{3}{|c|}{ Single-pole Cole-Cole } \\
\hline Adenoma HGD & 3.64 & 3.03 & 6.59 & 1.17 & 5.45 & 26.55 & 1.17 & 5.45 & 26.55 \\
\hline Adenoma LGD & 1.23 & 2.79 & 13.97 & 1.73 & 4.09 & 38.03 & 1.73 & 4.09 & 38.03 \\
\hline Hyperplastic & 38.91 & 1.57 & 4.32 & 37.86 & 1.73 & 5.25 & 37.86 & 1.73 & 5.25 \\
\hline
\end{tabular}

errors. The chi-squared values are in the range 1-5 and 1-14 for the relative permittivity and conductivity, respectively. For single-pole Cole-Cole model, the chi-squared values are slightly higher, indicating poorer data fitting to the model. The Cole-Cole fitting of our measurements provided very small values of $\alpha$ ranging from $1.5 \cdot 10^{-7}$ to 0.05 . Table $\mathrm{V}$ presents the single-pole Debye parameters of the five benign and malignant colon tissue groups derived from the median measured complex permittivity. Figure 10 depicts the resultant fits for the complex permittivity over $2.5-20 \mathrm{GHz}$ frequency band. The healthy colon mucosa has been compared to the data provided by Gabriel et al. ${ }^{33,53}$ showing better agreement for the conductivity rather than the relative permittivity.

\section{DISCUSSION}

We performed complex permittivity measurements on freshly excised colon samples of different types of polyps, cancer, and healthy mucosa tissues obtained from 23 patients from 0.5 to $20 \mathrm{GHz}$. After a sample selection process, we obtained 51 valid measurements. We discarded diminutive polyps and big ones resected in piecemeal fashion because they were too small to be measured reliably with our probe.
TABLE V. Single-pole Debye model parameters of benign and malignant colon tissues $(2.5-20 \mathrm{GHz}) . \epsilon_{\infty}$ is the infinite frequency permittivity, $\epsilon_{s}$ is the static permittivity, $\tau$ the relaxation time constant, and $\sigma_{s}$ the static ionic conductivity.

\begin{tabular}{lcccc}
\hline \hline Tissue & $\epsilon_{\infty}$ & $\epsilon_{s}$ & $\tau(\mathrm{ps})$ & $\sigma_{s}(\mathrm{~S} / \mathrm{m})$ \\
\hline Adenocarcinoma & 6.03 & 60.80 & 8.93 & 0.71 \\
Adenoma HGD & 6.49 & 50.14 & 7.36 & 1.04 \\
Adenoma LGD & 2.47 & 42.43 & 5.19 & 1.14 \\
Hyperplastic & 5.90 & 46.33 & 6.81 & 1.05 \\
Healthy mucosa & 5.05 & 44.79 & 5.62 & 1.23 \\
\hline \hline
\end{tabular}

A thinner probe would be more adequate for these samples. Although our measurements were performed ex vivo, we put a lot of effort in reducing the time between excision and measurement to get closer to the in vivo conditions. All measurements were acquired around 2 min after excision, except from cancer and healthy mucosa samples that were delayed up to $2 \mathrm{~h}$ (typically $30 \mathrm{~min}$ ). With the scarcity of data available in the literature, ${ }^{44,54-57}$ it is difficult to quantify how post-excision conditions can affect our measurements. There is a consensus in the literature ${ }^{56}$ that differences between 

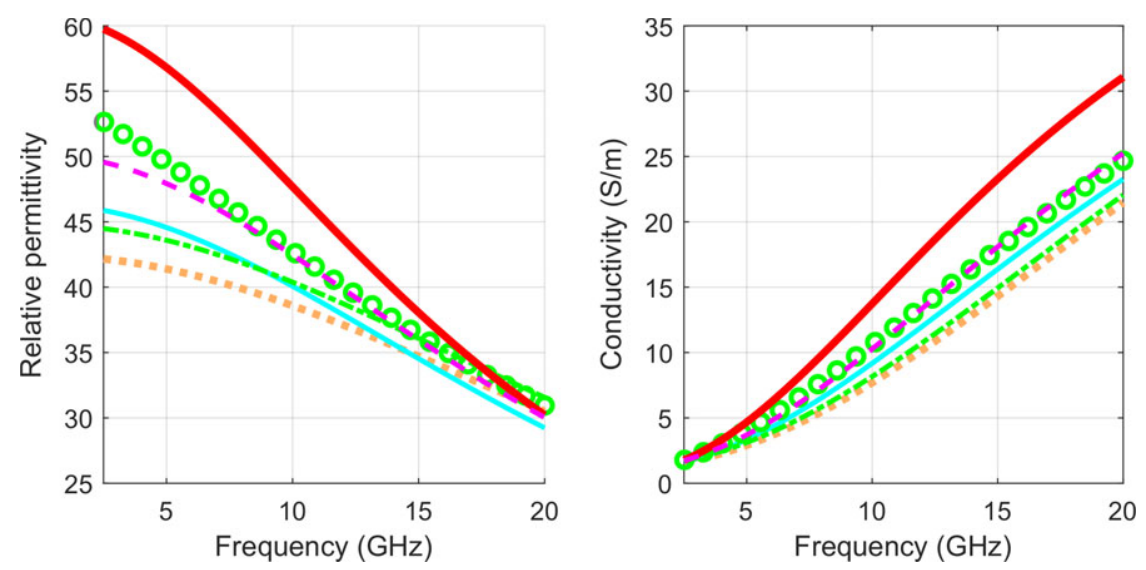

$$
\begin{array}{ll}
\hline \text { Adenocarcinoma } & =\text { Adenoma LGD } \\
-- \text { - Adenoma HGD } & \text { Hyperplastic }
\end{array}
$$

$==-=-$ Healthy mucosa

- Healty mucosa Gabriel et al.

FIG. 10. Single-pole Debye fits to the five categories of colorectal tissues. The healthy mucosa is compared to the available data in the literature (Gabriel et al. ${ }^{33}$ ). HGD and LGD stand for high-grade dysplasia and low-grade dysplasia, respectively. [Color figure can be viewed at wileyonlinelibrary.com]

in vivo and ex vivo properties are more important at low frequencies $(<100 \mathrm{MHz})$, also being quite dependent on the type of tissue. Haemmerich et al. ${ }^{54}$ measured swine liver resistivity (inverse of the conductivity) in vivo and after excision for $12 \mathrm{~h}$ and concluded that during the first $2 \mathrm{~h}$ postmortem (when temperature changes exist), resistivity increases by $32 \%$ at $1 \mathrm{MHz}$. In contrast, at high RF and microwave frequencies, the dielectric properties depend mainly on the water content and state (including temperature changes). Therefore, at higher frequencies, changes between in vivo and ex vivo properties of biological tissues can be minimized as long as the measurements are performed within a few hours after excision so that minimal liquid loss takes place. ${ }^{57}$ Nonetheless, a fair amount of controversy exists on the quantitation of this effect. O'Rourke et al. ${ }^{55}$ reported that the in vivo conductivity for normal tissue was $16 \%$ higher at $2.45 \mathrm{GHz}$ than the ex vivo conductivity, and that these changes were almost immediate. However, statistically significant differences were not found between the dielectric properties of in vivo and ex vivo malignant tissue. Another study ${ }^{44}$ reported no statistically significant changes into the complex permittivity of breast cancer tissues for times between excision and measurement smaller than $3 \mathrm{~h}$ and $29 \mathrm{~min}$. We also controlled the sample temperature, which due to the small size of the samples, rapidly arrived at room temperature (between 20 and $22^{\circ} \mathrm{C}$ ) and remained stable. Since no effect is expected due to a variation in temperature, ${ }^{44}$ this effect has not been studied further in this work.

The obtained measurements demonstrated that complex permittivity is well correlated with the dysplasia grade of polyps; higher values of the relative permittivity and conductivity in the examined tissue are linked to larger likelihood to be cancerous tissue. From a biophysical point of view, the predominant effect in the complex permittivity of human tissues between 0.5 and $20 \mathrm{GHz}$ is due to the orientation mechanism of water molecules (polarization). Therefore, the observed differences in complex permittivity reflect the difference in water content between cancer and normal colon tissues. In general, literature reports higher values of relative permittivity and conductivity for cancerous tissues than for normal tissues, due to the higher water content in the former ones. ${ }^{30}$ Thus, higher complex permittivity indicates higher tissue density, higher water content, and increasing cancer probability. ${ }^{26,57}$ In our case, two main mechanisms may be involved in elevating the water content of cancer and adenomas with HGD. First, increased mucus production in the tumor region may augment the extracellular water content. Tumor cells secrete mucus that invades the interstitial space producing large pools of liquid. ${ }^{58}$ Secondly, dysplasia is accompanied by angiogenesis and, therefore, greater vascular water content. This is consistent with the recent finding according to which the angiogenic switch occurs at the onset of the adenoma-carcinoma sequence in CRC..$^{59,60}$ As reported by Kristensen et al., ${ }^{61}$ the greatest increase in angiogenesis occurs at the earliest stage of dysplastic transformation. Another characteristic of the complex permittivity of adenocarcinomas is that the relative permittivity has a steeper slope, and the peak in the conductivity (possibly produced by the relaxation ${ }^{30}$ ) is shifted with respect to the other tissues. Recent studies relate complex permittivity with the grade of binding of water molecules present in tissues. ${ }^{26}$ The random molecular motion of water molecules can be quantitatively measured in vivo with diffusion-weighted magnetic resonance ${ }^{61-64}$ through the apparent diffusion coefficient (ADC). A low ADC value means more proportion of bound water and increased tissue density. Higher tissue density is related to more aggressiveness or metastatic capacity of tumors. Conversely, healthy tissues or benign pathological processes present high ADC values, indicating large extracellular space, less tissue density, and more water mobility. Studies in water solutions reflect that the complex permittivity of bound water resemble more to that of solid water that implies a shift of the relaxation to lower frequencies ${ }^{65}$ (the decay of the relative permittivity and the peak of the conductivity occurs at lower 
frequencies). Our results seem to be quite consistent with the previous reasoning: since malignant tissue has more bound water proportion than healthy colon tissue ${ }^{66,67}$ the relaxation of adenocarcinomas is shifted to lower frequencies with respect to benign tissue one.

Concerning the fitting results, we can observe a correlation between the static permittivity $\left(\epsilon_{s}\right)$ and tissue water content, ${ }^{32}$ and consequently, between $\epsilon_{s}$ and malignancy. The Cole-Cole model did not improve the results of the Debye model due to the negligible $\alpha$ values obtained. This may be due to the high water content of colon tissues, and would be consistent with Gabriel's results, ${ }^{32}$ in which body fluids reported negligible $\alpha$ values. Figure 10 shows that there is not very good agreement between our fitting of healthy mucosa and Gabriel's one. Our results indicate quite lower values of complex permittivity, which can be partly explained by the different temperatures and the different species between the two sets of measurements. Gabriel's data are from ovine colon samples acquired at $30^{\circ}$, while our data are from human colon samples at $20-22^{\circ}$. Joines et al. ${ }^{30}$ reported the complex permittivity in conditions more similar to ours (human colon samples at 23$25^{\circ}$ ) but in a lower frequency range (50-900 MHz). The values obtained by Joines et al. are also lower than Gabriel's data. Since most literature finds minimal differences between species, the different measurement temperatures could largely explain the discrepancies observed in Fig. 10. Another cause of fitting inaccuracy may be caused by the linear frequency spacing of the complex permittivity measurements. Some studies $^{31,32,51}$ show that linear spacing provided insufficient data points at lower frequencies in the Cole-Cole plots compared to measurements in the logarithmic scale, leading to less accurate fitting at frequencies $<1 \mathrm{GHz}$.

We reported the sensitivity and specificity to detect adenocarcinomas and different types of polyps with our method based on their complex permittivity values. The sensitivity at $5 \mathrm{GHz}$ is $100 \%$ for all lesions except for the adenoma with HGD that is $91 \%$. The specificity is $95 \%$ for the adenocarcinomas, $62 \%$ for the adenomas with HGD, $61 \%$ for the adenomas with LGD, and $74 \%$ for the hyperplastic polyps. By changing the threshold, it is possible to obtain a $100 \%$ value of sensitivity for the adenomas with HGD at the expense of reducing the specificity to $41 \%$. As a reference, conventional colonoscopy reports a sensitivity and specificity of 74-94\% and $88-94 \%$, respectively, to detect adenomas larger than $6 \mathrm{~mm} .{ }^{6}$ These values cannot be directly compared to the ones we obtain on ex vivo tissues since colonoscopy data are acquired in vivo. ${ }^{6}$ For having a fair benchmark, we should first have a complete microwave-based system operated by a physician in vivo such as a conventional colonoscopy. Colon angulations and folds, poor cleaning, and movements of the colon, among others, are some of the main challenges of colon imaging when performed in vivo.

We did not find statistical significant differences in dielectric properties due to the shape of the polyps. This may suggest that the complex permittivity could help to detect subtle (slightly elevated, sessile, or flat) polyps, which are the most difficult lesions to detect endoscopically. A study that tracked the performance of endoscopists in the detection of flat polyps reported low detection rates $(1.5-3.5 \%)$ during first 200 colonoscopies and $7.4 \%$ after 1000 colonoscopies. ${ }^{68}$ Flat lesions are also more likely to harbor advanced histology (HGD or early cancer) compared to pedunculated or sessile lesions irrespective of the size. Therefore, detection and subsequent removal of these lesions is of paramount importance for improving the efficacy of colonoscopy in preventing the development of CRC.

Aside from the detection itself, the proposed method adds in situ diagnosis capacity. With conventional colonoscopy, when a polyp is found, clinicians have to make decisions about resecting or not depending on the type of polyp and its risk to degenerate. This information is not available during colonoscopy and endoscopists only rely on subjective observations based on different surface or vascular patterns. The accurate diagnosis is only confirmed after excision with the pathology analysis. For this reason, the current protocol establishes to resect and analyze all lesions found during the exploration, which is time consuming and costly. The measurements presented in this paper, show the possibility to distinguish malignant tissues from healthy mucosa and benign colon tissues, since there is not overlapping between their complex permittivity below $10 \mathrm{GHz}$. Moreover, the grade of dysplasia can be inferred from the complex permittivity as it increases with dysplasia. Accordingly, polyps with LGD present a complex permittivity very close to that of the benign tissues and polyps with HGD occupy the intermediate region between healthy tissues and cancer.

\section{CONCLUSION}

In summary, we measured the complex permittivity of freshly excised healthy colon, different types of polyps, and colon cancerous tissues of human ex vivo samples between 0.5 and $20 \mathrm{GHz}$ from 23 patients. The obtained measurements, validated through pathological analysis, showed that the complex permittivity correlates with the grade of dysplasia of colon epithelium and thus can be used to quantify the grade of dysplasia or malignancy of the colon lesions. Our initial results reported a sensitivity of $100-91 \%$ and a specificity of $61-95 \%$ to detect adenocarcinomas, adenomas, and hyperplastic polyps from healthy mucosa. This study is the first demonstration of the feasibility of using complex permittivity data to provide complementary functional information to conventional colonoscopy. The development of a fully integrated multimodal (optical and microwave) colon imaging platform has the potential of making a positive impact of the management of CRC patients.

\section{ACKNOWLEDGMENTS}

This work was supported by the Department of Universitats, Recerca i Societat de la Informació of the Catalan Government through Llavor 2014LLAV00016 and producte 2016PROD00068 projects, La Caixa through Caixaimpulse program CI16-00058, the Spanish Comisión Interministerial 
de Ciencia y Tecnología (CICYT) under projects TEC201678028-C3-1-P, TEC2014-58582-R, the Spanish Agencia Estatal de Investigación Unidad de Excelencia Maria de Maeztu (MDM-2016-0600), the Spanish Ministerio de Economía, Industria y Competitividad, DTS17/00090 and CERCA Programme/Generalitat de Catalunya. The authors thank the Fundamental Physics Department of Universitat de Barcelona for their contribution to the complex permittivity measurements.

\section{CONFLICT OF INTEREST}

The authors have no conflicts to disclose. ${ }^{\text {a) }}$ Author to whom correspondence should be addressed. Electronic mail:
marta.guardiola@upf.edu.

\section{REFERENCES}

1. Parkin DM, Forman D, Bray F, et al. GLOBOCAN 2012 v1.0 Cancer Incidence and Mortality Worldwide. IARC CancerBase; 2013, 11. http://globocan.iarc.fr Acessed July 30, 2017.

2. GlobalData. PharmaPoint: Colorectal Cancer - Global Drug Forecast and Market Analysis to 2025; 2017.

3. Morillas JD, Castells A, Oriol I, et al. Alianza para la Prevención del Cáncer de Colon en España: un compromiso cívico con la sociedad. Gastroenterol Hepatol. 2012;35:109-128.

4. Fleming M, Ravula S, Tatishchev SF, Wang HL. Colorectal carcinoma: pathologic aspects. J Gastrointest Oncol. 2012;3:153-173.

5. Gonzalez-Pons M, Cruz-Correa M. Colorectal cancer biomarkers: where are we now? Biomed Res Int. 2015;2015:149014.

6. Lin JS, Piper MA, Perdue LA, et al. Screening for colorectal cancer: updated evidence report and systematic review for the us preventive services task force. JAMA. 2016;315:2576-2594.

7. Pox CP. Controversies in colorectal cancer screening. Digestion. 2014;89:274-281.

8. Müller AD, Sonnenberg A. Prevention of colorectal cancer by flexible endoscopy and polypectomy. A case-control study of 32,702 veterans. Ann Intern Med. 1995;123:904-910.

9. Winawer SJ, Zauber AG, Ho MN, et al. Prevention of colorectal cancer by colonoscopic polypectomy. N Engl J Med. 1993;329:1977-1981.

10. van Rijn JC, Reitsma JB, Stoker J, Bossuyt PM, van Deventer SJ, Dekker E. Polyp miss rate determined by tandem colonoscopy: a systematic review. Am J Gastroenterol. 2006;101:343-350.

11. Samadder NJ, Curtin K, Tuohy TM, et al. Characteristics of missed or interval colorectal cancer and patient survival: a population-based study. Gastroenterology. 2014;146:950-960.

12. Lee T, Rees C, Blanks R, et al. Colonoscopic factors associated with adenoma detection in a national colorectal cancer screening program. Endoscopy. 2014;46:203-211.

13. East JE, Saunders BP, Burling D, Boone D, Halligan S, Taylor SA. Surface visualization at CT colonography simulated colonoscopy: effect of varying field of view and retrograde view. Am J Gastroenterol. 2007;102:2529-2535.

14. Konda V, Chauhan SS, Abu Dayyeh BK, et al. Endoscopes and devices to improve colon polyp detection. Gastrointest Endosc. 2015;81:11221129.

15. Bernal J, Sánchez FJ, de Miguel CR, Fernández-Esparrach G. Building up the future of colonoscopy - a synergy between clinicians and computer scientists. In: Screening for Colorectal Cancer with Colonoscopy. London: InTech; 2015.

16. Manfredi MA, Abu-Dayyeh BK, Bhat YM, et al. Electronic chromoendoscopy. Gastrointest Endosc. 2015;81:249-261.

17. Mads SB, Wei Z, Kan L, et al. Characterizing variability of in vivo Raman spectroscopic properties of different anatomical sites of normal colorectal tissue towards cancer diagnosis at colonoscopy. Anal Chem. 2015;87:960-966.
18. Yu C, Fan S, Sun Y, Pickwell-MacPherson E. The potential of terahertz imaging for cancer diagnosis: a review of investigations to date. Quant Imaging Med Surg. 2012;2:33-45.

19. Bolomey JC, Jofre L. Three Decades of Active Microwave Imaging Achievements, Difficulties and Future Challenges. Honolulu, HI: IEEE ICWITS. 2010;2010:1-4.

20. Lazebnik M, Popovic D, McCartney L, et al. A large-scale study of the ultrawideband microwave dielectric properties of normal, benign and malignant breast tissues obtained from cancer surgeries. Phys Med Biol. 2007;52:6093-6115.

21. Winter A, Laing J, Paglione R, Sterzer F. Microwave hyperthermia for brain tumors. Neurosurgery. 1985;17:387-399.

22. Nagae D, Mase A. Measurement of heart rate variability and stress evaluation by using microwave reflectometric vital signal sensing. Rev Sci Instrum. 2010;81:94301.

23. Semenov SY, Corfield DR. Microwave tomography for brain imaging: feasibility assessment for stroke detection. Int J Antennas Propag. 2008;2008:1-8.

24. Meaney PM, Goodwin D, Golnabi AH, et al. Clinical microwave tomographic imaging of the calcaneus: a first-in-human case study of two subjects. IEEE Trans Biomed Eng. 2012;59:3304-3313.

25. Grzegorczyk TM, Meaney PM, Kaufman PA, di Florio-Alexander RM, Paulsen KD. Fast 3-D tomographic microwave imaging for breast cancer detection. IEEE Trans Med Imaging. 2012;31:15841592.

26. Meaney PM, Golnabi AH, Epstein NR, et al. Integration of microwave tomography with magnetic resonance for improved breast imaging. Med Phys. 2013;40:103101.

27. Preece AW, Craddock I, Shere M, Jones L, Winton HL. MARIA M4: clinical evaluation of a prototype ultrawideband radar scanner for breast cancer detection. J Med Imaging. 2016;3:33502.

28. Done-Sik Y, Bong-Seok K, Hyung-Do C, Ae-Kyoung L, Jong-Ki P. The dielectric properties of carcinomas. In: Proceedings of the XXVIIth URSI General Assembly; 2002.

29. Done-Sik Y. The dielectric properties of cancerous tissues in a nude mouse xenograft model. Bioelectromagnetics. 2004;25:492-497.

30. Joines WT, Zhang Y, Li C, Jirtle RL. The measured electrical properties of normal and malignant human tissues from 50 to $900 \mathrm{MHz}$. Med Phys. 1994;21:547-550.

31. Gabriel S, Lau RW, Gabriel C. The dielectric properties of biological tissues: II. Measurements in the frequency range $10 \mathrm{~Hz}$ to $20 \mathrm{GHz}$. Phys Med Biol. 1996;41:2271-2269.

32. Gabriel S, Lau RW, Gabriel C. The dielectric properties of biological tissues: III. Parametric models for the dielectric spectrum of tissues. Phys Med Biol. 1996;41:2271-2293.

33. Andreuccetti D, Fossi D, Petrucci C. An Internet resource for the calculation of the dielectric properties of body tissues in the frequency range $10 \mathrm{~Hz}-100 \mathrm{GHz}$. IFAC-CNR. http://niremf.ifac.cnr.it/tis sprop/.

34. Kumar V, Stanley L. Robbins Basic Pathology, 8th ed. Philadelphia, PA: Saunders/Elsevier; 2007.

35. Morson B. The polyp-cancer sequence in the large bowel. Proc R Soc Med. 1974;67:451-457.

36. Rex DK, Ahnen DJ, Baron JA. Serrated lesions of the colorectum: review and recommendations from an expert panel. Am J Gastroenterol. 2012;107:1315-1330.

37. Bauer VP, Papaconstantinou HT. Management of serrated adenomas and hyperplastic polyps. Clin Colon Rectal Surg. 2008;21:273-279.

38. The Paris endoscopic classification of superficial neoplastic lesions: esophagus, stomach, and colon. Gastrointest Endosc. 2003;58:23-43.

39. Kim NH, Jung YS, Jeong WS, et al. Miss rate of colorectal neoplastic polyps and risk factors for missed polyps in consecutive colonoscopies. Intest Res. 2017;15:411-418.

40. Keysight 85070 E Dielectric Probe Kit $200 \mathrm{MHz}$ to $50 \mathrm{GHz}$ Technical Overview. Keysight Technologies, United States; 2017. http://literature. cdn.keysight.com/litweb/pdf/5989-0222EN.pdf.

41. No K, Hilland J, Kaatze U. Dielectric properties of aqueous $\mathrm{NaCl}$ solutions at microwave frequencies. 1997;101:6864-6869.

42. Gregory AP, Clarke RN. Tables of the complex permittivity of dielectric reference liquids at frequencies up to $5 \mathrm{GHz}$. NPL Report, United Kingdom; 2012. 
43. Riley SA. Colonoscopic Polypectomy and Endoscopic Mucosal Resection: a Practical Guide. United Kingdom: British Society of Gastroenterology; 2008.

44. Martellosio A, Pasian M, Bozzi M, et al. Dielectric properties characterization from 0.5 to $50 \mathrm{GHz}$ of breast cancer tissues. IEEE Trans Microw Theory Tech. 2017;65:998-1011.

45. Von Hippel A. Dielectric Materials and Applications. Norwood, MA: A. House; 1954.

46. Mustafa S, Abbosh AM, Nguyen PT. Modeling human head tissues using fourth-order Debye model in convolution-based three-dimensional finite-difference time-domain. IEEE Trans Antennas Propag. 2014;62:1354-1361.

47. Lazebnik M, McCartney L, Popovic D, et al. A large-scale study of the ultrawideband microwave dielectric properties of normal breast tissue obtained from reduction surgeries. Phys Med Biol. 2007;52: 2637-2656.

48. O'Halloran M, Conceicao RC, Byrne D, Glavin M, Jones E. FDTD modeling of the breast: a review. Prog Electromagn Res B. 2009;18:124.

49. Foster KR, Schepps JL, Schwan HP. Microwave dielectric relaxation in muscle. A second look. Biophys J . 1980;29:271-281.

50. Schepps JL, Foster KR. The UHF and microwave dielectric properties of normal and tumour tissues: variation in dielectric properties with tissue water content. Phys Med Biol. 1980;25:1149-1159.

51. Salahuddin S, Porter E, Meaney PM, O'Halloran M. Effect of logarithmic and linear frequency scales on parametric modelling of tissue dielectric data. Biomed Phys Eng Express. 2017;3:1-22.

52. Florkowski CM. Sensitivity, specificity, receiver-operating characteristic (ROC) curves and likelihood ratios: communicating the performance of diagnostic tests. Clin Biochem Rev. 2008;29:S83-S87.

53. Gabriel C, Gabriel S. Compilation of the Dielectric Properties of Body Tissues at RF and Microwave Frequencies Internet document. Retrieved from: http://www.dtic.mil/dtic/tr/fulltext/u2/a305826.pdf.

54. Rossmann C, Haemmerich D. Review of temperature dependence of thermal properties, dielectric properties, and perfusion of biological tissues at hyperthermic and ablation temperatures. Crit Rev Biomed Eng. 2014;42:467-492.
55. O'Rourke AP, Lazebnik M, Bertram JM, et al. Dielectric properties of human normal, malignant and cirrhotic liver tissue: in vivo and ex vivo measurements from 0.5 to $20 \mathrm{GHz}$ using a precision open-ended coaxial probe. Phys Med Biol. 2007;52:4707-4719.

56. Allen TH, Krzywicki HJ, Roberts JE. Density, fat, water and solids in freshly isolated tissues. J Appl Physiol. 1959;14:1005-1008.

57. Foster KR, Schwan HP. Dielectric properties of tissues and biological materials: a critical review. Crit Rev Biomed. 1989;17:197-211.

58. Kufe DW. Mucins in cancer: function, prognosis and therapy. Nat Rev Cancer. 2009;9:874-885.

59. Staton CA, Chetwood ASA, Cameron IC, Cross SS, Brown NJ, Reed MWR. The angiogenic switch occurs at the adenoma stage of the adenoma carcinoma sequence in colorectal cancer. Gut. 2007;56:14261432.

60. Raica M, Cimpean AM, Ribatti D. Angiogenesis in pre-malignant conditions. Eur J Cancer. 2009;45:1924-1934.

61. Kristensen CA, Askenasy N, Jain RK, Koretsky AP. Creatine and cyclocreatine treatment of human colon adenocarcinoma xenografts: $31 \mathrm{P}$ and $1 \mathrm{H}$ magnetic resonance spectroscopic studies. $\mathrm{Br} \mathrm{J}$ Cancer. 1999;79:278-285.

62. Kilickesmez O, Atilla S, Soylu A, et al. Diffusion-weighted imaging of the rectosigmoid colon. J Comput Assist Tomogr. 2009;33:863-866.

63. Tomoaki I, Sukru ME, Motosugi U, et al. High-B-value diffusionweighted MRI in colorectal cancer. Am J Roentgenol. 2006;187:181184.

64. Jianfeng W, Kan L, Wei Z, et al. Fiber-optic Raman spectroscopy for in vivo diagnosis of gastric dysplasia. Faraday Discuss. 2016;187:377392.

65. Kaatze U. Bound water: evidence from and implications for the dielectric properties of aqueous solutions. J Mol Liq. 2011;162:105-112.

66. Pivarnik JM, Palmer RA. Water and Electrolytes During Exercise. (Hickson JF, Wolinski I, eds.). Boca Raton; CRC Press; 1994.

67. Milo R, Jorgensen P, Moran U, Weber G, Springer M. BioNumbersthe database of key numbers in molecular and cell biology. Nucl Acids Res. 2010;38:750-753.

68. Rastogi A. Clinical review: how to recognize subtle lesions in the colon. ASGE Lead Edge. 2015;5:1-12. 